\title{
The experience of tertiary care public hospital in Oman: Lean performance improvement to enhance universal health coverage
}

\author{
Jehan Al Fannah, Ismail Al Rashdi, Harith Al Harthy, Qasem Al Salmi \\ Royal Hospital, Oman
}

Received: June 28, 2019

Accepted: September 9, 2019

Online Published: September 16, 2019

DOI: $10.5430 /$ jha.v8n5p52

URL: https://doi.org/10.5430/jha.v8n5p52

\begin{abstract}
Healthcare systems at an organizational level need to equip its people with tools that ensure continuous performance improvement to help sustain Universal Health Coverage (UHC). A large tertiary care public hospital in Oman (Royal Hospital [RH]), is sharing its experience in performance improvement using lean methodology. The RH introduced lean performance improvement to improve the efficiency of its administrative and clinical processes. This remarkable change was a cultural shift as it empowered clinical and non-clinical teams to improve the performance of the hospital operations continuously. Hospital leaders need to create opportunities for sustainable UHC at an organizational level. Introducing lean improvement methods at the hospital level leads to better problem definition and resolution. The people who do the work, need to improve the work, and eliminate waste in the processes as a day to day work style.
\end{abstract}

Key Words: Lean hospital, Oman health, Lean method, Universal health coverage, Lean performance improvement

\section{INTRODUCTION}

Achieving universal health coverage (UHC) by 2030 is embedded in the sustainable development goals (SDGs), and is a work program priority for the World Health Organization (WHO) over the next decade. ${ }^{[1]}$ The WHO defines UHC as all individuals and communities receiving the health services they need without suffering financial hardship, with coverage for the full spectrum of essential, quality health services, from health promotion to prevention, treatment, rehabilitation, and palliative care. ${ }^{[2]}$ The 2017 WHO report, shows Oman having the full spectrum of UHC similar to Eastern Europe. ${ }^{[3]}$ However, the global economic crisis is negatively impacting countries with reliance on oil production and fluctuating oil prices; such as Oman. ${ }^{[4]}$ Gulf Co-operation Council countries of which Oman is a member, are among the fastest- growing populations in the world. Although Oman has a low burden of infectious diseases, it has a notable increase in non-communicable diseases. ${ }^{[5]}$ The chronic nature of noncommunicable diseases is an epidemiological challenge to UHC sustainability, creating a need for "continuous service improvement". ${ }^{[6]}$ There's an international agreement on the importance of implementing and sustaining UHC. However, in the celebration of the 40th anniversary of the Alma Ata Declaration in 2018, there was a call to reconsider the role of public hospitals and specialized care in the primary healthcare system. ${ }^{[7]}$ More focus is needed to understand hospital reform integration into the broader health system reform, especially in the context of global prioritization of quality and equity in access to UHC. ${ }^{[7]}$ Harnessing innovation in healthcare (e.g., in pharmaceuticals, diagnostics, devices)

*Correspondence: Jehan Al Fannah; Email: jehan.alfannah@gmail.com; Address: Royal Hospital, Oman. 
judiciously, and in information and communication technologies could substantially change health service provision. ${ }^{[8]}$ The experience of the Royal Hospital (RH) in performance improvement augments the recent report shared by the WHO, about hospitals being powerful with influential impact as institutions that have political, economic and social weight to make change happen. ${ }^{[9]}$

Oman has a population of 4.6 million (NCSI, 2019). The RH is a tertiary care public hospital. It is the largest medical institute (970 beds) in the country and operates autonomously with a fully integrated health information system (HIS), that connects it with the rest of the Ministry of Health Institutes. The RH provides "free access" to healthcare and its main specialties are surgery, medicine, obstetrics and gynecology, child health, emergency medicine, nuclear medicine, and other supportive services. In addition, to an oncology center, heart center, genetics center, endocrine center, and hyperbaric medicine center. It is the country's lead provider for kidney and liver transplantation, home ventilation, and home total parenteral nutrition. Oman has a homogenous healthcare system with access to primary healthcare and an e-referral system to higher-level care such as secondary and tertiary care. The local healthcare system challenges are similar to global healthcare systems, such as increased demand and delayed access to higher-level care. There is a shortage of care providers, fears of not enough surgeons, doctors, and nurses to treat people. Despite all that, healthcare systems are full of waste and experience an enormous amount of variation. $^{[10]}$

Lean performance improvement is a continuous, incremental, and measurable improvement, which originated in the car industry. ${ }^{[11]}$ It is believed to have an essential role in healthcare operations by tackling multiple challenges that plaque the healthcare systems. Lean methods enhance the elimination of various system wastes such as over-processing, overproduction, underutilized talent, inventory, motion, transportation, waiting-time, and defects. Also, some tools define the value-added step in the patient journey. ${ }^{[12]}$ Hence, in any service that faces bottlenecks in the process, it is essential to explore the eight wastes. It is equally important to value stream map (VSM) the process from the client's perspective. The implementation of the $5 \mathrm{~s}$ lean method improves environmental efficiency. Lean improves care provider contribution to service efficiency and timeliness. ${ }^{[13,14]}$ Although lean performance improvement methods have been used in multiple high-income countries such as the USA and the UK for more than a decade now, there is little literature to describe its use outside the European Continent and the USA. ${ }^{[14]}$ Therefore, the primary focus of this article is to share some of the methods used to improve hospital performance while reflecting

Published by Sciedu Press on its potential to enhance UHC.

\section{SetTing AND Methods}

In September 2017 to March 2018, the hospital leadership invested in introducing awareness and training program on improvements through lean methodology, facilitated by a hired external consultant. He visited the hospital monthly for seven months. The participants exercised, as part of the curriculum, application of lean in multiple improvement projects. Lean application tools such as the A3-problem solving tool, value stream mapping of processes (VSM), and the application of $5 \mathrm{~S}$. These tools led to the build-up of projects as the lean champions established regular monthly internal workshops. There were bi-monthly awareness presentations on lean in the main hospital auditorium throughout the year.

However, there were multiple other initiatives, as illustrated in Figure 1. The main aim of the lean improvement program was building capacity and capability through the initial development of 32 lean champions. Lean champions continue to lead the change in their clinical areas while advocating and training in lean throughout the hospital as culture stewards. Focus on lean was further supported and facilitated by the establishment of a performance improvement unit (PIU), led by a senior care provider with an advanced management degree. The change was further enhanced and steered through leadership support (see Table 1). Site visits by top management became opportunities for leaders to meet frontline teams, listen to them, and acknowledge their work. The visit emphasized the value of reducing the distance between the layers of the organization hierarchy. Also, these were opportunities to share and learn from the successful improvements and failures, modeling values of a learning organization. It is an opportunity to share and acknowledge successful improvements. Hospital leadership visits to site of action (GEMBA Walk) to "see the work", "ask why" and "show respect" these are some of the fundamental principles of lean improvements and leadership commitment.

\section{ReSUlts}

\subsection{Lean performance improvement}

At an organizational level, lean improvement methods used influenced the hospital culture change. Two hundred improvement projects (see Figure 2) reported, 81.5\% (163) completed by the end of 2018. Projects related to environmental efficiency and the application of $5 \mathrm{~S}$ represented $41.5 \%$ (83) of the projects. Projects related to operational efficiency and access represented 34\% (68). Projects impacting quality and safety represented $24.5 \%$ (49). There were several other lean improvement projects ongoing in various departments, but not necessarily reported to PIU. There were 14 
workshops conducted on lean methods by the champions in their departments or through the Training Department with an average of 22-29 attendance rate per workshop. Many of the improvement projects (see Table 2) had an impact on the service timeliness by reducing the waiting time, reschedul- ing or cancellation rate, and inventory control. Departmental and interdepartmental engagement led to an average of 400 employees trained in the first year on lean methods. Over 16 leadership GEMBA walks happened during the same period.

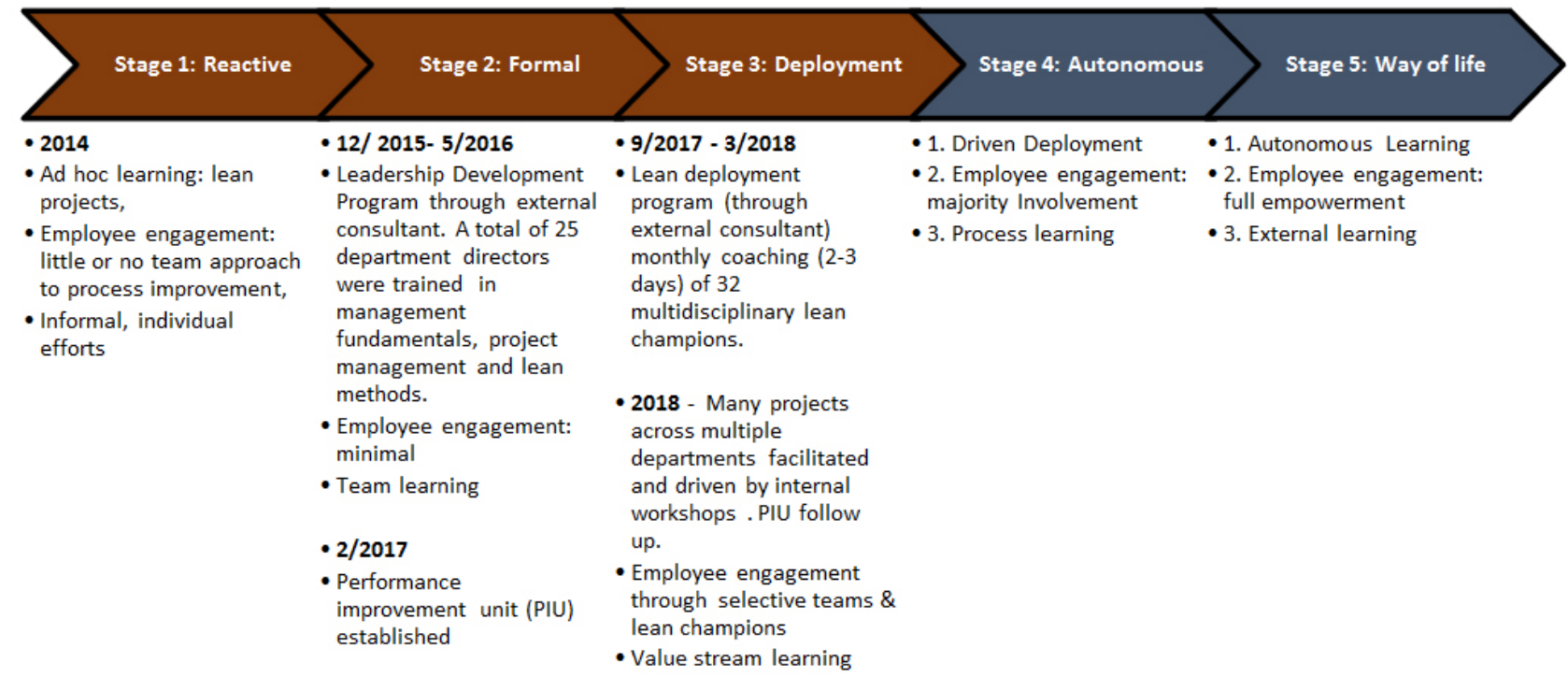

Figure 1. The RH Lean Maturity (Lean Business System $®$ )

Table 1. Leadership support summary to enhance lean culture maturity

Performance Improvement Steering Committee (PISC) - monthly

Chaired by the hospital CEO or his Deputies

Members (20-25)

All Clinical/Service Directors/PIU leader

Ad Hoc Non-Clinical Directors, e.g., Finance, IT, Health Information

Lean Champions

Benefits

Contributes to consistency of progress in continuous improvement

\section{Leadership GEMBA Walk - biweekly}

Attendance

Benefits

Establishing Performance Improvement Unit (PIU)

Benefits
Hospital CEO and his deputies

Encouraged: clinical directors/front line providers/lean champions

Enhances top-down and bottom-up communication

Follow up progress

Syncronize strategic directions

Create an agenda for PISC according to feedback and progress

Participate in creating awareness \& training

Encourage social media presence for successful projects, and leadership GEMBA

Unification of efforts and building of data for hospital improvements 


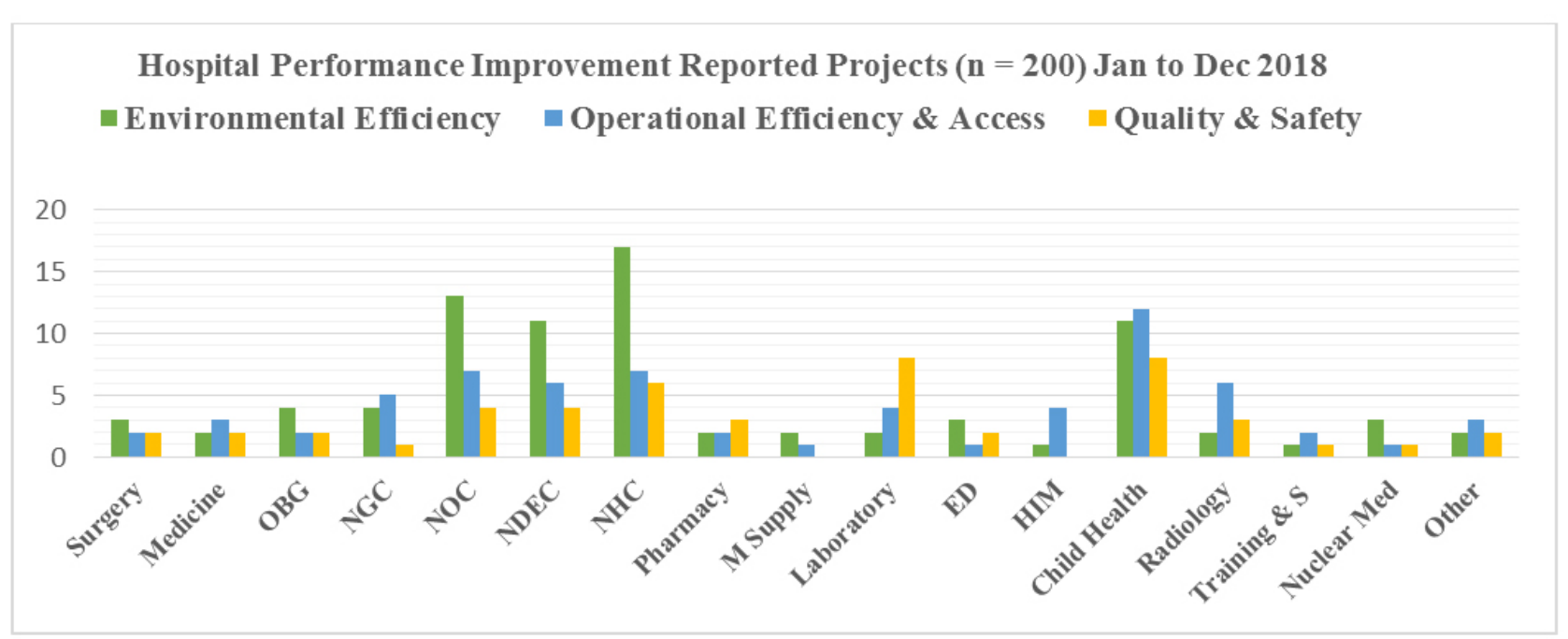

Figure 2. Hospital performance improvement projects $(\mathrm{n}=200)$ using lean methods

Table 2. Examples of projects according to category

\begin{tabular}{|c|c|}
\hline Category & Outcome (2018) \\
\hline Environmental Efficiency & $\begin{array}{l}\text { - 5s application produced } 127 \mathrm{~m}^{2}(\sim 40,000) \text { of space across the hospital (indirect cost } 1 \mathrm{~m}^{2}= \\
\$ 300) \text { March - September } 2018 \text {. } \\
\text { - Filtering of equipment (not used and donated) contributed to the creation of space. }\end{array}$ \\
\hline Elimination of Waste (in \$) & $\begin{array}{l}\text { - Return of unused inventory from the various wards for re-stocking or utilization in other } \\
\text { units }(\sim 230,000) \text {. }\end{array}$ \\
\hline Operational Efficiency \& Access & $\begin{array}{l}\text { - Pediatric oncology day care reduced waiting at the bed for the administration of } \\
\text { chemotherapy from } \sim 120 \text { minutes to } \sim 30 \text { minutes (2018). Improved bed utilization. } \\
\text { - VSM application reduced surgical cancellation rate from } 17 \% \text { to } 5 \% \text { over three months } \\
\text { (2018). } \\
\text { - Re-arrangement of ward storage space. Reduced time wasted looking. Variable time saving } \\
\text { of } \sim 2-4 \text { min per item fetched per nurse (2018). }\end{array}$ \\
\hline Quality \& Safety & $\begin{array}{l}\text { - } 5 \mathrm{~s} \text { use resulted in the availability of areas for safe medication preparation at the ward level, } \\
\text { which reduced medication errors at the bedside. }\end{array}$ \\
\hline
\end{tabular}

\section{Discussion}

\subsection{Lean performance improvement}

The hospital is at stage three of the lean mature organization (see Figure 1). The application of lean at the RH has contributed to the improvement of the timeliness of processes. Also, lean had an impact on reducing the hierarchy and creating more effective team-based work with the empowerment of multiple professions, especially the nursing teams. The nurse empowerment is a very well described effect in the literature. ${ }^{[15]}$ There is an emerging literature to support the finding that lean methods create a positive psychosocial environment when employees are directly involved in the change process. ${ }^{[16]}$ Furthermore, leadership support, including the regular top management visits to the sites of action contributes to the change in organizational culture. However,

Published by Sciedu Press changing leadership behaviors, such as curiosity, humility, and reflection, are directly linked to lean culture sustainability. It takes time to develop such behaviors across all the different leadership levels. There were critical success factors for the hospital during the deployment and implementation phase in the RH that have been backed up by the literature, such as gaining stakeholder buy-in, effective vertical and horizontal communication. ${ }^{[17]}$ Some of the projects needed information system adjustments to enhance process sustainability. An external consultant is essential at the beginning to create a sense of urgency. However, measurable change occurs through a shift in culture and leadership behavior, not only by introducing the implementation team with improvement methods. Internationally, research is still ongoing, on how lean improvements are affecting patients' experience, 
professional practice, and healthcare systems. ${ }^{[18,19]}$

\subsection{Enhancing universal health coverage}

The WHO uses 16 essential health services in four categories as indicators of the level and equity of health coverage. The categories are maternal, and newborn care, infectious diseases, non-communicable diseases, and the fourth category is service capacity and access. ${ }^{[20]}$ The reported project categories described in Figure 2, such as environmental efficiency, access, and operational efficiency, quality, and safety all have a direct or indirect impact on healthcare system reform at an organizational level. The fourth category of UHC indicators is where lean improvement methods can be used to enhance UHC.

\subsection{Practice implications and challenges for healthcare managers}

Leadership support summarized in Table 1, show practice implications for healthcare managers. However, it is equally important to note here, not unexpectedly, the hospital witnessed change management difficulties. For example, some departments tended to revert away from the lean improvement approach for various reasons, such as the set of priorities in a busy working environment that did not align with the hospital goals. Furthermore, some lean champions with mounting clinical responsibilities made it difficult to commit to coaching on lean methods. Hence, exploring the value of creating "protected time" for lean influential practitioners should be considered, especially in the early years of a lean organization. There may be a need to integrate improvements with a well-defined career development pathway and a reward system.

\section{Conclusions}

There is a crucial need for hospitals to improve their environmental efficiency, timely and efficient access to care with high quality and enhanced patient safety. Lean performance improvement methods prepare care providers with ways that help define the problem from a client perspective. The lean improvement approach and outcomes described here may positively enhance the sustainability of UHC at an organizational level.

\section{Limitations}

The lean performance improvement methods used may require more operational research in developing countries to verify its impact on timely access to UHC.

\section{ACKNOWLEDGEMENTS}

Lean champions, health care providers, and especially nursing teams for their contribution to the hospital's continuous performance improvement.

\section{CONFlicts OF INTEREST Disclosure}

The authors declare they have no conflicts of interest.

\section{REFERENCES}

[1] Hogan DR, Stevens GA, Hosseinpoor AR, et al. Monitoring universal health coverage within the Sustainable Development Goals: development and baseline data for an index of essential health services. The Lancet Global Health. 2018 Feb 1; 6(2): e152-68. https://doi.org/10.1016/S2214-109X (17)30472-2

[2] Boerma T, Eozenou P, Evans D, et al. Monitoring progress towards universal health coverage at country and global levels. PLoS medicine. 2014 Sep 22; 11(9): e1001731. PMid: 25243899. https : //doi.org/10.1371/journal.pmed.1001731

[3] World Health Organization. Tracking universal health coverage: 2017 global monitoring report. 2017. Available from: https://apps. who.int/iris/bitstream/handle/10665/2 59817/9789241513555-eng.pdf ; jsessionid=DD8DF80B97 4E675618082BFDE065BEAA? sequence $=1$

[4] Young KE. The Difficult Promise of Economic Reform in the Gulf. 2018. Available from: https: //www .bakerinstitute.org/media/files/files/a9b 497ad/cme-pub-carnegie-young-092618.pdf

[5] Khoja T, Rawaf S, Qidwai W, et al. Health care in Gulf Cooperation Council countries: a review of challenges and opportunities. Cureus 2017 Aug; 9(8). PMid: 29062618. https://doi.org/10.7759/ cureus. 1586
[6] McKee M, Balabanova D, Basu S, et al. Universal health coverage: a quest for all countries but under threat in some. Value Health. 2013; 16(1 Suppl): S39-45. PMid: 23317643. https: //doi.org/10.1016/j.jval.2012.10.001

[7] Chabrol F, Albert L, Ridde V. 40 years after Alma-Ata, is building new hospitals in low-income and lower-middle-income countries beneficial? BMJ Global Health. 2019 Apr 1; 3(Suppl 3): e001293. PMid: 31168419. https://doi.org/10.1136/bmjgh-2018-001293

[8] Bloom G, Katsuma Y, Rao KD et al. Next steps towards universal health coverage call for global leadership. BMJ. 2019; 365: 12107. PMid: 31126926. https://doi .org/10.1136/bmj . 12107

[9] WHO Technical Series on Primary Care. The transformative role of hospitals in the future of primary health care. 2018. Available from: https://www. who.int/docs/default-source/primary-h ealth-care-conference/hospitals. pdf?sf vrsn=5d7e813 7_2

[10] Inozu B, Chauncey D, Kamataris V, et al. Performance improvement for healthcare: Leading change with lean, six sigma, and constraints management. New York: McGraw-Hill; 2012.

[11] Moraros J, Lemstra M, Nwankwo C. Lean interventions in healthcare: do they actually work? A systematic literature review. International journal for quality in health care. 2016 Jan 24; 28(2): 150-65. PMid: 26811118. https://doi.org/10.1093/intqhc/mzv123 
[12] Goodridge D, Rana M, Harrison EL, et al. Assessing the implementation processes of a large-scale, multi-year quality improvement initiative: a survey of health care providers. BMC health services research. 2018 Dec; 18(1): 237. PMid: 29615014. https: //doi.org/10.1186/s12913-018-3045-6

[13] Aij KH, Simons FE, Widdershoven GA, et al. Experiences of leaders in the implementation of Lean in a teaching hospital-barriers and facilitators in clinical practices: a qualitative study. BMJ Open. 2013 Oct. PMid: 24171938. https : //doi.org/10.1136/bmjopen-2 013-003605

[14] Terra JD, Berssaneti FT. Application of lean healthcare in hospital services: a review of the literature (2007 to 2017). Production. 2018; 28. https://doi.org/10.1590/0103-6513.20180009

[15] Mason J, Wesorick B. Successful transformation of a nursing culture. Nurse Leader. 2011 Apr 1; 9(2):31-6. https ://doi.org/10.101 $6 / j \cdot \mathrm{mnl} .2011 .01 .001$

[16] Ulhassan W, von Thiele Schwarz U, Thor J, et al. Interactions between lean management and the psychosocial work environment in a hospital setting-a multi-method study. BMC Health Services Re- search. 2014 Dec; 14(1): 480. PMid: 25339236. https://doi.or g/10.1186/1472-6963-14-480

[17] Noori B. The critical success factors for successful lean implementation in hospitals. International Journal of Productivity and Quality Management. 2015 Jan 1; 15(1): 108-26. https://doi.org/10.1 504/IJPQM. 2015.065987

[18] Woodnutt S. Is lean sustainable in today's NHS hospitals? A systematic literature review using the meta-narrative and integrative methods. International Journal for Quality in Health Care. $2018 \mathrm{Apr}$ 10; 30(8): 578-86. PMid: 29648651. https://doi.org/10.109 3/intqhc/mzy070

[19] Rotter T, Adegboyega L, Fiander M, et al. Lean management in health care: effects on patient outcomes, professional practice, and healthcare systems. The Cochrane Database of Systematic Reviews. 2017 Nov; 2017(11). https://doi.org/10.1002/14651858.C D012831

[20] World Health Organization. Universal Health Coverage. 2019, January 24. Available from: https ://www . who. int/news-room/fa ct-sheets/detail/universal-health-coverage-(uhc) 